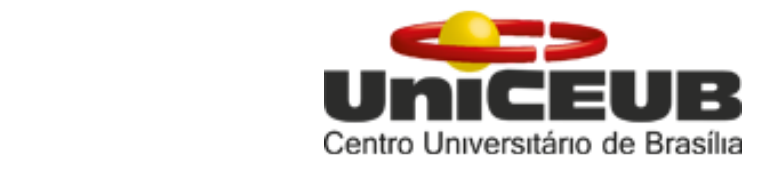

CENTRO UNIVERSITÁRIO DE BRASÍLIA - UniCEUB

PROGRAMA DE INICIAÇÃO CIENTÍFICA

MARIA GABRIELLY MACÊDO COSTA

VALIDAÇÃO DO PROTOCOLO DE MANEJO AMBIENTAL PARA CONTROLE DE LUTZOMYIA LONGIPALPIS EM ÁREAS ENDÊMICAS PARA

LEISHMANIOSE VISCERAL

BRASÍLIA 


\section{Unलि= \\ Centro Unıversıtárıo de Brasílıa}

\section{MARIA GABRIELLY MACÊDO COSTA}

VALIDAÇÃO DO PROTOCOLO DE MANEJO AMBIENTAL PARA CONTROLE DE LUTZOMYIA LONGIPALPIS EM ÁREAS ENDÊMICAS PARA LEISHMANIOSE VISCERAL

Relatório final de pesquisa de Iniciação Científica apresentado à Assessoria de Pós-Graduação e Pesquisa

Orientação: Rafaella Albuquerque e Silva

BRASÍLIA 


\title{
VALIDAÇÃO DO PROTOCOLO DE MANEJO AMBIENTAL PARA CONTROLE DE LUTZOMYIA LONGIPALPIS EM ÁREAS ENDÊMICAS PARA LEISHMANIOSE VISCERAL
}

\author{
Maria Gabrielly Macêdo Costa - UniCEUB, PIC Institucional, aluno \\ bolsista \\ gabrielly.costa@sempreceub.com
}

Rafaella Albuquerque e Silva- UniCEUB, professor orientador rafaella.silva@ceub.edu.br

A leishmaniose visceral (LV) é uma antropozoonose causada pelo protozoário Leishmânia infantum e transmitida pelo flebotomíneo Lutzomyia longipalpis. Essa doença vem modificando seus padrões de transmissão e adquirindo um caráter urbano, periurbano e reemergente nos últimos anos. Os novos padrões epidemiológicos de transmissão conjugam inúmeros fatores relacionados à população humana, a população de vetores, de reservatórios, bem como as condições ambientais. Partindo desse pressuposto, torna-se importante elaborar medidas que acompanhem os novos padrões da doença, a partir da intervenção epidemiológica e ações de educação em saúde, visando oferecer a população informações acerca da transmissão, prevenção e controle da LV. Dessa forma, 
esse estudo objetiva validar o protocolo de manejo ambiental para controle da população de flebotomíneos de Brasília, Distrito Federal. Portanto, foi selecionado para participar do estudo o Condomínio Rancho Karina (RK), localizado em Sobradinho, devido à ocorrência de casos humanos de LV em 2014 e a prevalência de LV canina de 9,75\% em 2016. Foi realizada uma amostragem probabilística, onde foram selecionados 322 domicílios do condomínio. A primeira etapa do projeto consiste na visita aos domicílios para a realização da classificação dos imóveis quanto ao risco de ocorrência de LV; aplicação dos questionários quanto à percepção da população a respeito das medidas de prevenção e controle preconizadas para os vetores da LV; coleta de sangue para realização do protocolo de diagnóstico de LV canina; realização da investigação entomológica e aplicação do protocolo de manejo ambiental que deve ser seguido pelos moradores. A segunda etapa do projeto consistirá no retorno a todos os domicílios amostrados anteriormente para reavaliação das condições ambientais, da prevalência de LV canina, da presença de vetores e do conhecimento da população sobre a doença. Até o momento, foram amostradas 40 residências, entretanto somente 37 foram classificadas: 4 (11\%) como de alto risco, 22 (59\%) de médio risco, 10 (27\%) de baixo risco e 1 (3\%) sem risco. Foram testados 63 animais, sendo 11 (17,5\%) positivos na triagem, a partir do TR-DPP, entretanto espera-se o resultado do Elisa, teste confirmatório. No tocante a mensuração do conhecimento da população, $68 \%$ das pessoas sabiam o que era LV e qual o seu agente etiológico, $90 \%$ sabiam que ela é uma doença transmitida por vetores, entretanto somente $26 \%$ sabiam quem é o principal vetor transmissor. O condomínio RK, pontuando principalmente as características ambientais, é considerado uma área de risco para a transmissão de leishmaniose visceral, com $97 \%$ das casas visitadas consideradas receptivas ao vetor e com 17\% de prevalência da doença em cães. Mesmo vivenciando este contexto, a população não está sensibilizada para a ocorrência da doença. Mediante estes resultados, é importante a manutenção das atividades no condomínio RK, para que seja finalizada a primeira etapa do projeto, e assim obtido os dados necessários para a comparação na segunda etapa. Isto permitirá ter uma avaliação do protocolo de manejo ambiental para controle de Lutzomyia longipalpis. 
Palavras-Chave: Leishmaniose visceral. manejo ambiental. Lutzomyia longipalpis. cães. 


\section{SUMÁRIO}

1 INTRODUÇÃO

2. OBJETIVOS

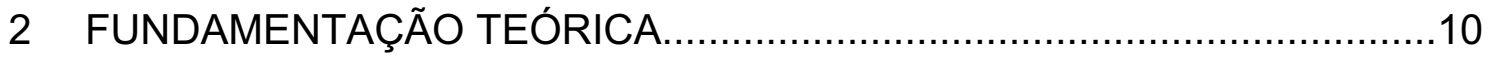

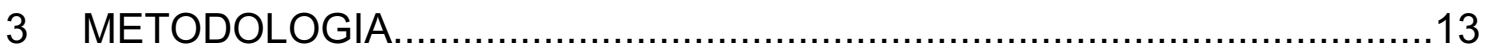

$4 \quad$ RESULTADOS E DISCUSSÃO...........................................................19

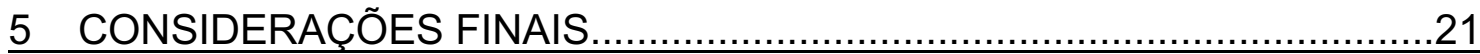

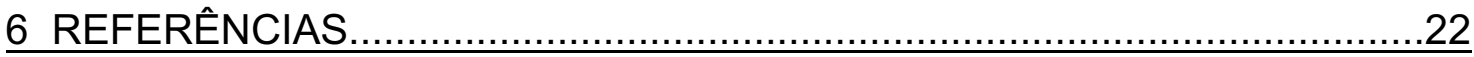

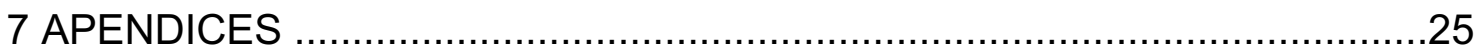




\section{INTRODUÇÃO}

Atualmente, observa-se o aumento de doenças reemergentes que deixam o país em uma situação de alerta. Uma destas é a leishmaniose visceral (LV), que anteriormente era um problema exclusivamente silvestre e na atualidade tem sido motivo de preocupação em áreas urbanas também. Isso devido a modificação dos padrões de transmissão desta doença, o que conjugou fatores relacionados à população humana, de reservatórios, de vetores, bem como às condições ambientais.

Descrita pela primeira vez nas Américas em 1913 e em 1936 no Brasil, é possível observar, a partir de uma série histórica, que os casos de leishmaniose visceral vêm aumentando no Brasil, principalmente em grandes centros urbanos (CONTI, R. V.; et al, 2016).

O estudo realizado por Conti et al (2016) apresenta a leishmaniose visceral dividida em três fases de acordo com o contexto histórico do país. A primeira fase se fez entre 1930 a 1950, descrita como endêmica do meio rural. A segunda fase vai até a década de 80 , com o primeiro caso urbano descrito. Já a terceira fase é caracterizada pela consolidação da transmissão endêmica urbana, com um exacerbado número de casos nas cidades brasileiras.

No tocante a epidemiologia da doença, pode-se observar que ela está distribuída por todas as regiões brasileiras, tendo sido notificados casos em 23 unidades federativas. Em 2017, foram confirmados 4300 casos da doença, sendo este o maior pico de casos desde 2012. Vale ressaltar que, algumas regiões, anteriormente indenes a doença, apresentaram casos nos últimos anos. É o caso do Paraná e Santa Catarina, que apresentaram casos humanos em 2014 e 2018 respectivamente (Sinan/SVS/MS, 2018).

Todas as idades são acometidas pela leishmaniose visceral, porém no país cerca de $80 \%$ dos casos registrados até 2011 foram em crianças com menos de 10 anos (NALEVAIKO et al, 2011). Atualmente, este percentual reduziu para $47 \%$. Entretanto, apesar da redução deste percentual, quando analisada a letalidade da doença, esta é maior nos extremos de idade (menores de um ano e maiores de 50 anos).

O impacto produzido pela leishmaniose na saúde da população humana, devido a alta incidência, letalidade e implicações econômico-sociais dão à esta enfermidade uma alta importância política e social. As leishmânias, agentes 
causadores das leishmanioses, podem ser divididas em dois grupos distintos, porém ambos importantes e complexos. Um grupo causador da leishmaniose tegumentar (LT), que compreendem espécies como Leishmania braziliensis, Leishmania amazonenses, Leishmania guyanensis dentre outros; e um outro da leishmaniose visceral (LV), compreendendo a espécie Leishmania infantum (MONTEIRO, 2017).

Os vetores responsáveis pela transmissão destes agentes etiológicos causadores das leishmanioses são os flebotomíneos do gênero Lutzomyia, conhecidos popularmente como mosquito-palha. Estes têm predileção por repasto sanguíneo em galinhas, mas o principal reservatório urbano é o cão (TAYLOR; COOP; WALL, 2016).

Os hospedeiros definitivos da leishmaniose são os humanos e os cães, em um ambiente urbano, e ainda as raposas, os lobos, guaxinins, rato-preto, entre outros vindos de um ambiente silvestre. Os cães, novamente, são de grande importância na manutenção do ciclo da doença em área urbana, devido ao fato de que neles a carga parasitária periférica é muito alta. Sabe-se que os casos humanos são precedidos pelos casos caninos, e dessa forma, os cães podem ser considerados também animais sentinelas para a ocorrência da doença em humanos (TAYLOR; COOP; WALL, 2016).

A partir da picada das fêmeas infectadas dos flebotomíneos, os hospedeiros são infectados. As formas imaturas dos vetores desenvolvem-se em ambientes ricos em matéria orgânica e com baixa densidade luminosa. Ele pode ser encontrado na forma promastigota no canal alimentar dos vetores e na forma amastigota no fígado, baço e medula óssea dos hospedeiros vertebrados (MONTEIRO, 2017).

É possível observar tanto animais assintomáticos quanto animais clinicamente positivos, com sinais que vão desde áreas de alopecia a caquexia. Por ser uma doença crônica, tais sinais podem levar de meses a anos para surgirem (TAYLOR; COOP; WALL, 2016).

O Programa de Controle da LV do Ministério da Saúde do Brasil (PVCLV) tem como objetivos a diminuição do número de óbitos da doença e a redução da taxa de letalidade. O sucesso dos objetivos supracitados permanece como um grande desafio dado a insustentabilidade da realização das atividades preconizadas de forma continuada e integral, principalmente em áreas com alta densidade populacional (PEIXOTO et al., 2014; SALOMÓN et al., 2015; 
ROMERO, 2016). Dessa forma, estudos com objetivo de validar novas ferramentas devem ser estimulados. E é isso que se propõe o presente estudo.

\section{OBJETIVO GERAL}

Validar o protocolo de manejo ambiental para controle da população de flebotomíneos, comparando áreas submetidas à intervenção com áreas sem esta intervenção, em municípios endêmicos para leishmaniose visceral no Brasil.

\section{OBJETIVOS ESPECÍFICOS}

1. Comparar a frequência de Lu. longipalpis nos ambientes de coleta: intra e peridomicílio, entre áreas com a realização do manejo ambiental e áreas sem esta intervenção;

2. Calcular a abundância relativa de Lu. longipalpis por residência comparando este índice em áreas com a realização do manejo ambiental e áreas sem esta intervenção;

3. Calcular a taxa de infestação domiciliar de Lu. longipalpis por residência comparando esta taxa em áreas com a realização do manejo ambiental e áreas sem esta intervenção;

4. Calcular o coeficiente de prevalência de LV em cães comparando esta taxa em áreas com a realização do manejo ambiental e áreas sem esta intervenção;

5. Mensurar o conhecimento da população residente em áreas endêmicas acerca da leishmaniose visceral, medidas de prevenção e controle;

6. Trabalhar a percepção social das leishmanioses nas comunidades de municípios endêmicos para leishmaniose visceral;

7. Desenvolver ações de Educação e Saúde, como medida de prevenção e controle da leishmaniose visceral; 


\section{FUNDAMENTAÇÃO TEÓRICA}

De acordo com Silva et all (2008), as doenças causadas por protozoários do gênero Leishmania foram consideradas como uma das doenças tropicais mais importantes pela Organização Mundial da Saúde (OMS). No Brasil, distribuída em todas as suas regiões está o principal transmissor da leishmaniose visceral, o Lutzomyia longipalpis. Vale ressaltar que estudos mais recentes mostram a competência vetorial de outras espécies de flebotomíneos na transmissão da L. infantum. São eles a Lutzomyia cruzi e Lutzomyia migonei.

No tocante a severidade da doença, estudo realizado no Iraque por AlHamash (2012), mostra que dois a cada três doentes tinham menos de 2 anos de idade, semelhante ao que ocorre no Brasil. Esse fato pode ser justificado devido a imaturidade do sistema imunológico da criança, o que limita o estabelecimento de uma resposta imune efetiva. Ademais, neste mesmo estudo foi observado que o maior número de casos foi durante o inverno e a primavera, isto acorda com a relação da época reprodutiva do vetor ser no verão, com climas mais tropicais. O Brasil, por não possuir as quatro estações anuais bem definidas, sendo que somente o inverno e o verão são intensos, apresenta clima tropical favorável para esta reprodução vetorial quase o ano todo, com exceção dos estados mais ao sul que possuem inverno acentuado.

Considerando a forma de transmissão da LV, o controle vetorial é recomendado pelo Ministério da Saúde, entretanto estudos ainda mostram a dificuldade de identificar as formas imaturas de flebotomíneos no campo, o que limita a utilização do controle químico somente para as formas adultas do vetor e dessa forma, prejudica a efetividade desta atividade (READY et al., 2014).

Ainda sobre o estudo de Ready (2014), coleiras inseticidas, sprays e rollons são usados de maneira ampla no sul da Europa, para oferecer uma proteção aos cães, entretanto, sem ter sido confirmada a redução da transmissão para os humanos até então. Dentre as atividades preconizadas, a vigilância entomológica, o controle químico vetorial e o manejo ambiental são aquelas voltadas para a população de vetores. A vigilância entomológica é composta por três atividades: investigação, levantamento e monitoramento entomológico, cada uma com objetivos e indicações distintas dependentes da classificação epidemiológica da área. Estas atividades direcionam as áreas para a realização do controle químico vetorial. 
O controle químico, por sua vez, é uma medida voltada para a saúde coletiva e consiste na aplicação continuada de inseticidas de ação residual (borrifação) nos domicílios das áreas com casos confirmados de LV. Esta medida tem uma série de limitações operacionais a começar pela complexa metodologia de aplicação, necessidade de número elevado de agentes de endemias para execução, custo e cooperação dos proprietários das áreas com casos da doença (CAMARGO-NEVES et al., 2004; MORAIS et al., 2015; ZUBEN \& DONALÍSIO, 2016).

Trabalhos demonstram grande percentual de recusa da borrifação pelos moradores, devido à preparação do domicílio para a atividade bem como a necessidade de permanência fora dos domicílios durante um tempo após a realização da borrifação (DIAS, 1998; TAUIL, 2006; SILVA et al., 2007; SANTANA-FILHO, 2012; MORAIS et. al. 2015). Camargo-Neves (2004) relata dificuldades similares no estado de São Paulo, Brasil, principalmente em áreas urbanas, com alta densidade demográfica, o que faz com que a manutenção das atividades ao logo do tempo se torne inviável.

A falta de consentimento dos moradores à prática da borrifação muitas vezes está relacionado a falta de informação sobre a doença, seus riscos e meios de controle. Nesta situação, resta ao poder público orientar o morador quanto às práticas de manejo ambiental e os riscos advindos da não borrifação do imóvel, incorporando também estratégias focadas na conscientização da população.

Assim, a educação em saúde seria, portanto, atividade essencial para solucionar este problema e aumentar a eficiência das ações de controle da doença. O conhecimento sobre a doença do nível político para as comunidades é essencial para uma abordagem adequada à prevenção e controle desta doença. A disposição e o empenho político, bem como a cooperação intersetorial entre ministérios e agências são cruciais para garantir o sucesso das ações de controle (OMS, 2010).

A participação da comunidade no controle de Lu. longipalpis envolve um processo educacional que visa encorajar tal participação de uma forma que melhore e integre o conhecimento popular em suas práticas. A educação em saúde permite a formação de noções profundamente fundadas sobre a transmissão da doença, o preenchimento de lacunas na compreensão de seus hábitos e conhecimento dos lugares preferidos para viver em condições 
favoráveis ao seu desenvolvimento; este novo conhecimento pode contribuir para a prevenção e controle.

O manejo ambiental, por sua vez, é uma ferramenta que visa reduzir o contato homem-vetor e, assim, novos casos da doença em questão. Mudanças ambientais como limpeza, remoção de resíduos orgânicos, poda de árvores e redução de fontes de umidade são medidas que impedem o desenvolvimento das formas imaturas do vetor, uma vez que estas necessitam de matéria orgânica, temperatura e umidade. Neste contexto, sugere-se que há um impacto direto na curva populacional de vetores na área em que esta atividade é aplicada, o que demonstra que essa pode ser uma ferramenta positiva para populações expostas ao risco de adquirir LV (DEANE, 1956, LAINSON \& RANGEL 2005, CAMARGO-NEVES et al., 2007; ROMERO et al., 2016). Entretanto faltam evidências da sua efetividade, bem como protocolo validado para o seu desenvolvimento.

A pesquisa sobre a determinação social do risco de LV e a atuação efetiva dos indivíduos para cumprir as recomendações do sistema de saúde, além da construção social e percepção do risco por cada grupo social envolvido, também contribuem para a contextualização do problema. Portanto, a perspectiva sistêmica transdisciplinar, unindo a contextualização biológica e social, é uma abordagem essencial para promover a participação comunitária além da colaboração comunitária. Esta participação implica pensar em conjunto com a comunidade sobre as medidas mais apropriadas para o meio ambiente. 


\section{METODOLOGIA}

\section{1. ÁREA DE ESTUDO}

Os critérios utilizados para seleção das áreas de estudo se basearam nos dados epidemiológicos de LV humana e canina, disponíveis no sistema de informação de agravos de notificação (SINAN) e planilhas paralelas das equipes de vigilância epidemiológica do município de Brasília, DF.

Foram elencadas as áreas com maior concentração de casos de LV humana e canina e que apresentem características favoráveis ao aparecimento do vetor, tais como: presença de unidades domiciliares com amplo peridomicílio, presença de animais domésticos, acúmulo de matéria orgânica vegetal e animal, dentre outros. Neste sentido, foi escolhido o condomínio Rancho Karina (RK), localizado em Sobradinho, pois o último caso autóctone notificado no DF teve como local provável de infecção este condomínio, além de ter uma prevalência canina acima de $15 \%$.

\subsection{INDICADORES DE MORBIDADE}

Incidência da LV humana

Para a avaliação da LV humana foram utilizados os dados relativos aos casos confirmados notificados no Sistema de Informação de Agravos de Notificação (SINAN) durante um período de dez anos, oriundos da Coordenação de Vigilância Epidemiológica da Secretaria Municipal de Saúde do Governo do Distrito Federal.

A população humana do município em estudo a ser utilizada nos cálculos dos índices de incidência será obtida nos bancos de dados do IBGE e suas projeções.

Prevalência da LV canina

Os dados relativos à infecção canina no mesmo período, foram originados da demanda passiva e de busca ativa (inquéritos caninos) junto ao Diretoria de Vigilância Ambiental (Dival), vinculado à Secretaria Municipal de Saúde. O coeficiente de prevalência foi calculado considerando os casos confirmados no período, disponíveis nas bases de dados do município. 


\subsection{INTERVENÇÃO}

Foi realizada a classificação dos imóveis quanto ao risco de transmissão da LV, as atividades de manejo ambiental (Apêndice A) e aplicação de questionário (Apêndice $\mathrm{C}$ ).

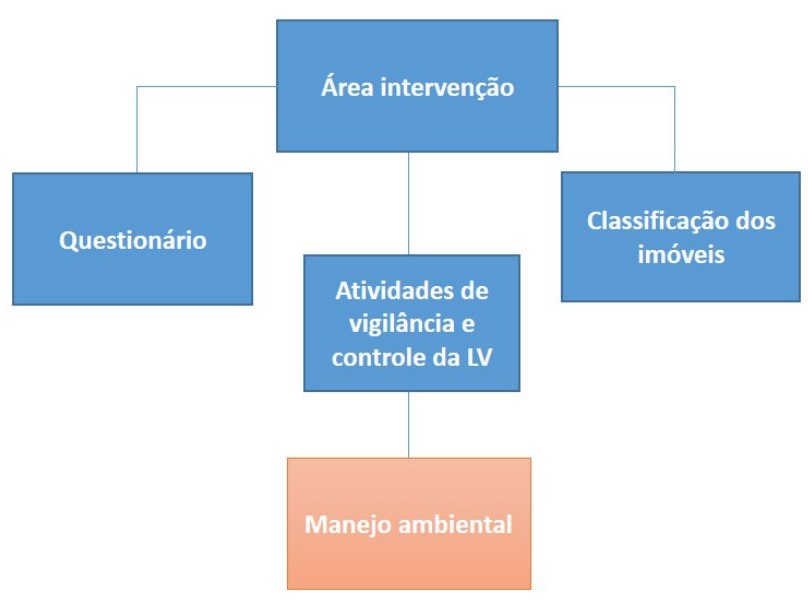

Figura 1. Fluxograma das atividades desencadeadas nas áreas de intervenção para validação do protocolo de manejo ambiental para controle da população de flebotomíneos em áreas endêmicas para leishmaniose visceral.

4.4. Classificação dos imóveis

Inicialmente os imóveis serão classificados quanto à presença de características ambientais, seguindo os parâmetros descritos no quadro abaixo:

Quadro 1. Classificação dos imóveis considerando parâmetros ambientais favoráveis ao desenvolvimento de vetores.

\begin{tabular}{|c|c|}
\hline Classificação do Imóvel & Parâmetros \\
\hline \multirow[t]{2}{*}{ Alto Risco } & $\begin{array}{l}\text { Peridomicílio de tamanho maior ou igual ( } \geq \text { ) a 200m2 com } \\
\text { presença de vegetação (árvores frutíferas ou } \\
\text { ornamentais), sombreamento e umidade; Acúmulo de } \\
\text { matéria orgânica em decomposição (lixo orgânico) } \\
\text { associado a presença de animais. }\end{array}$ \\
\hline & $\begin{array}{l}\text { Peridomicilio de tamanho maior ou igual ( } \geq \text { ) a 200m2 com } \\
\text { presença de vegetação (árvores frutíferas ou } \\
\text { ornamentais), sombreamento e umidade; Acúmulo de } \\
\text { matéria orgânica em decomposição (lixo orgânico). }\end{array}$ \\
\hline Médio Risco & $\begin{array}{l}\text { Presença de um ou mais dos seguintes fatores: } \\
\text { Acúmulo de folhas e frutos no chão }\end{array}$ \\
\hline
\end{tabular}




\begin{tabular}{|l|l|}
\hline & $\begin{array}{l}\text { Amontoado de tijolos, telhas e madeiras } \\
\text { Presença fezes para adubação (esterco). }\end{array}$ \\
\hline Baixo Risco & Sem nenhuma das condições acima \\
\hline Sem Risco & Quintal todo cimentado \\
\hline
\end{tabular}

Posteriormente a classificação dos imóveis, foi repassado ao morador às mudanças ambientais necessárias (Apêndice A), exclusivamente para os moradores da área de intervenção. $O$ imóvel será reavaliado a cada três meses para acompanhar as atividades de manejo ambiental.

\subsection{Manejo ambiental}

As atividades de manejo foram realizadas pela população da área de intervenção a partir das recomendações sobre o protocolo e manejo ambiental fornecidas pelos membros do projeto de pesquisa (Apêndice $A$ ), e com o devido consentimento dos moradores das residências inseridas no estudo por meio da assinatura do TCLE.

As recomendações de manejo ambiental incluem as providências a serem adotadas pelos moradores em relação ao peridomicílio, cuidados de higiene e saúde dos animais domésticos que sejam domiciliados nas suas respectivas residências. $O$ protocolo inclui também a definição se a residência vai passar pela pesquisa entomológica e a classificação do mesmo enquanto ao risco de aparecimento e/ou manutenção do vetor da LV (Apêndice A).

\subsection{Aplicação do questionário}

Para análise da percepção da população a respeito das medidas de prevenção e controle preconizadas para os vetores da LV serão realizadas entrevistas com a população.

Os entrevistados foram os residentes da área do estudo. Somente um membro de cada residência participou. O morador foi previamente informado sobre os objetivos e metodologia geral da pesquisa e foi convidado a assinar o termo de consentimento livre e esclarecido (Apêndice B). Apenas os moradores que concordarem com a pesquisa assinando o termo foram incluídos no estudo.

Estas entrevistas foram orientadas por meio de um questionário estruturado (Apêndice C) contendo perguntas de cunho objetivo a respeito da 
doença, formas de transmissão, vetores e agente etiológico, bem como sobre as medidas relativas à prevenção e controle da LV, como em relação às atividades de vigilância entomológica e controle químico desempenhada pelo município, medidas de prevenção individuais e coletivas, bem como posição e postura do proprietário frente à realização das atividades de controle.

Para tanto, o número de indivíduos que foram entrevistados neste estudo foi estimado o tamanho da amostra para se estimar uma Proporção (p) de população finita (MARTINS; THEÓPHILO, 2009) a partir da seguinte fórmula:

$\mathrm{n}=\quad \mathrm{z}^{2} \cdot \mathrm{p} \cdot \mathrm{q} \cdot \mathrm{N}$

$$
d^{2}(N-1)+z^{2} \cdot \text { p. q }
$$

Onde:

$\mathrm{N}=$ tamanho da população.

$Z=$ abscissa da curva normal padrão - com o valor de 1,96.

$p=$ estimativa da proporção $-0,5$.

$q=1-p-0,5$.

$\mathrm{d}=$ erro amostral, sendo utilizado 0,05.

$\mathrm{n}=$ tamanho da amostra aleatória simples a ser selecionada pela população.

\subsection{Investigação Entomológica}

As capturas serão realizadas em três setores da área. Em cada setor foram escolhidos três pontos de coleta (domicílios) para a realização da captura, com uma distância mínima de 200 metros entre eles. Para a escolha do domicílio foram consideradas características sugestivas a presença do vetor, tais como: vegetação abundante, animais domésticos e acúmulo de matéria orgânica. Ademais, foram priorizados domicílios com histórico de aparecimento de Lutzomyia longipalpis, caso esta informação esteja disponível.

Coletas de flebotomíneos

Em cada um dos setores amostrados no estudo foram selecionadas quatro residências de cada área onde serão colocadas armadilhas para captura 
de flebotomíneos seguindo metodologia preconizada pelo Ministério da Saúde (BRASIL, 2006).

Os moradores de cada domicílio serão informados sobre os objetivos e metodologia geral da pesquisa e, aceitando participar, assinarão o Termo de Consentimento Livre e Esclarecido - TCLE (Apêndice D), na qual será marcada a data do primeiro dia de colocação da armadilha.

Para a captura dos insetos serão utilizadas armadilhas luminosas tipo $\mathrm{CDC}$ miniatura, as quais serão instaladas às $18 \mathrm{~h} 00 \mathrm{~min}$ e retiradas às $06 \mathrm{~h} 00 \mathrm{~min}$ no intra e peridomicilio das residências previamente escolhidas, com distância de $1 \mathrm{~m}$ acima do solo e em ambientes com pouca iluminação. A coleta ocorrerá durante três noites consecutivas a cada três meses e terá duração mínima de 12 meses.

Considerando a importância dos fatores climáticos sobre as populações do vetor, no momento da captura serão anotadas informações de temperatura e umidade com o auxílio de um termo higrômetro, para avaliação das variáveis ambientais: temperatura $\left({ }^{\circ} \mathrm{C}\right)$ e umidade relativa do ar $(\%)$ referentes ao período de estudo.

Diariamente, após a retirada das armadilhas, as mesmas serão armazenadas em recipientes resfriados e transportadas para o Labocien, sob responsabilidade do estudante do PIC e da orientadora do projeto. No laboratório será realizada inicialmente a triagem, separando os flebotomíneos dos demais insetos. Os flebotomíneos serão colocados separados de acordo com o local e captura, mês de coleta, sexo e armazenados em tubos tipo eppendorf contento álcool isopropílico.

Identificação de flebotomíneos

Os flebotomíneos coletados e triados serão identificados de acordo com a classificação proposta por Galati (2003).

\section{AÇÕES DE EDUCAÇÃO EM SAÚDE}

A prática educativa tem como objetivo o repasse de informações sobre a LV, sua vigilância e controle. E será planejada de forma que o saber popular seja valorizado. Assim, pretende-se realizar a prática de educação em saúde durante a aplicação dos questionários, pesquisa entomológica e classificação dos imóveis, ou seja, durante as visitas às residências ao longo de todo o estudo. 


\section{ANÁLISE DOS DADOS}

O cálculo dos indicadores de morbidade (incidência de casos humanos e prevalência de casos caninos) utilizou as seguintes fórmulas:

Prevalência canina: Número de casos caninos no ano $\mathrm{x}$, na área y Pop. canina no mesmo período e local

Incidência humana: _Número de novos casos humanos no ano x, na área y Pop. humana no mesmo período e local

Serão utilizadas as seguintes fórmulas para o cálculo da taxa de infestação domiciliar (1) e distribuição destas espécies por sítio de coleta (2), respectivamente:

1) Total de domicílio positivo por espécie/local pesquisado/técnica $\times 100$

$$
\mathrm{N}^{\circ} \text { de local pesquisado }
$$

2) $\quad \mathrm{N}^{\circ}$ de Lu. longipalpis coletados por metodologia nos sítios de coleta (intra e peri) Total de sítios pesquisados por metodologia (intra e peri)_

A avaliação da distribuição da espécie vetora de LV e a correlação das frequências com o ambiente de coleta serão analisados utilizando o programa estatístico GraphPad InStat software 5.0.

As informações obtidas pelo questionário serão organizadas em tabelas de excel e feita uma análise descritiva sobre o conhecimento prévio e posterior às atividades desenvolvidas na comunidade para prevenção e controle da LV, em especial ao controle do vetor.

SUBMISSÃO AO COMITÊ DE ÉTICA: Todo o transcurso da Pesquisa será desenvolvido respeitando os princípios Éticos preconizados pelas com as Resoluções n 466/12 e 510/16 do Conselho Nacional de Saúde do Ministério da Saúde. Os procedimentos relacionados às entrevistas e entomologia foram 
submetidos à aprovação do Comitê de Ética em Pesquisa, e possuem a aprovação n. 016252/2018.

\section{RESULTADOS E DISCUSSÃO}

O trabalho de campo foi realizado de maneira conjunta com os profissionais do próprio condomínio. No condomínio RK existe o Centro de Estudos Ambientais (CEA), que realiza atividades de educação em saúde e mobilização social para todas as doenças mais frequentes no DF, além de realizar a busca de foco de Aedes aegypti casa a casa.

Mesmo quando nos domicílios não havia animais, era realizada a classificação dos imóveis, a sensibilização do morador quando à doença e o trabalho de pesquisa que está sendo realizado, assim como eram repassadas as recomendações a cerca do protocolo de manejo ambiental. Não foi possível terminar a primeira etapa do projeto, devido ao atraso na entrega dos materiais que seriam utilizados em campo.

Até o momento, somente foram amostradas 40 residências, entretanto somente 37 foram classificadas considerando as características ambientais, sendo 4 (11\%) classificadas como de alto risco, 22 (59\%) como de médio risco, $10(27 \%)$ como de baixo risco e 1 (3\%) como sem risco. Dentre as características mais comumente observadas, a presença de árvores frutíferas e matéria orgânica foram as mais comuns. Todas as casas amostradas tinham cães como animais domésticos.

No período de estudo foram testados 63 animais, sendo 11 positivos na triagem, a partir do TR-DPP, entretanto estão esperando o resultado do Elisa, teste confirmatório. O Elisa é realizado no Laboratório de Diagnóstico de Leishmaniose Visceral Canina da Diretoria de Vigilância Ambiental, DIVAL, DF. Caso seja comprovada a positividade, a prevalência canina amostral será de $17 \%$.

No tocante a mensuração do conhecimento da população, $68 \%$ das pessoas sabiam o que era LV e qual o seu agente etiológico, 90\% sabiam que ela é uma doença transmitida por vetores, entretanto somente $26 \%$ sabiam quem é o principal vetor transmissor. Esses resultados indicam o desconhecimento a cerca do vetor, o que mostra que as pessoas podem estar convivendo com os mesmos sem saber. Da mesma forma, é fundamental a sensibilização da 
população quanto as medidas de proteção individual, para a interrupção da transmissão tanto nos animais como nos humanos.

Observa-se, em estudos semelhantes sobre o conhecimento da população, que de modo geral as pessoas não detêm de informações necessárias para entender a transmissão da doença, fato indispensável no controle da mesma. Segundo Menezes et al (2016), que realizou um estudo no município de Formiga, Minas Gerais, o nível de informação das pessoas sobre a LV é baixo, além do fato delas, em sua maioria, viverem em situação de "risco peridomiciliar", pois vivem em condições insalubres, com muita matéria orgânica em decomposição, o que contribui para a manutenção da doença nesta área do município.

Já no estado de São Paulo percebe-se um conhecimento maior quanto a LV e seus sintomas, pois são realizadas atividades de divulgação. Vale enfatizar ainda que, mesmo em situação favorável, é sugerida uma melhor divulgação das práticas preventivas quanto à doença e um apoio maior do governo $\mathrm{e}$ colaboração da população (Anvesa, 2016).

Tradicionalmente, estão inseridas em nos programas de controle de doenças sob vigilância no Brasil, ações de educação em saúde. Alguns autores ainda, como Zuben \& Donalísio (2016), relatam as dificuldades encontradas para se implementar um programa de vigilância e controle da leishmaniose visceral quanto mais para se manter as alterações implementadas, seja por falta de recursos ou de colaboração humana. Todos os estudos que envolvem participação externa, seja com ações humanas ou patrocínios, exigem uma atenção maior em sua manutenção, o que não será diferente na validação para o protocolo de manejo ambiental para o controle de Lutzomyia longipalpis.

Ainda não é possível uma descrição a cerca da investigação entomológica com a coleta de flebotomíneos, pois estas ações ainda serão realizadas, assim como para com a prevalência tanto dos casos caninos como dos casos humanos. 


\section{CONSIDERAÇÕES FINAIS}

A LV é uma importante zoonose que, a cada ano se expande mais no país, evidenciando a ineficiência das atividades de controle tradicionais. Neste sentido, a busca por outras alternativas é uma realidade atual.

As áreas estudadas são áreas favoráveis para o desenvolvimento e manutenção da doença, uma vez que foram referidas presença de reservatórios domésticos, vetores e histórico de tutores que perderam seus animais com leishmaniose visceral.

O nível de conhecimento a cerca da doença ultrapassou a média até então, mas para não se ter nenhum viés de informação é de grande relevância que o projeto dê continuidade para que se tenha os resultados com todas as amostras.

Algumas limitações deste estudo podem ser destacadas, como as recusas de alguns proprietários em participar do estudo, bem como as casas que no momento da visita estavam fechadas.

Vale ressaltar que as atividades a campo foram postergadas devido ao atraso na compra dos materiais para o inquérito sorológico e captura dos flebtomíneos, o que impediu a obtenção de todos os resultados necessários para concluir-se os objetivos do projeto.

Como conclusão evidenciamos a importância da manutenção das atividades no condomínio RK, para que seja finalizada a primeira etapa do projeto, e assim obtido os dados necessários para a comparação na segunda etapa. Isto permitirá ter uma avaliação do protocolo de manejo ambiental para controle de Lutzomyia longipalpis. 


\section{REFERÊNCIAS}

Al-Hamash, Sadiq. Study of visceral leishmaniasis (kala-azar) in children of Iraq. Mustansiriya Medical Journal, 2012.

ANVERSA, L.; MONTANHOLI R.J.D.; SABINO D.L. Avaliação do conhecimento da população sobre leishmaniose visceral. Rev Inst Adolfo Lutz. São Paulo, 75:1685; 2016.

BRASIL, Ministério da Saúde. Manual de vigilância e controle da leishmaniose visceral. Secretaria de Vigilância em Saúde. Departamento de Vigilância Epidemiológica. Editora do Ministério da Saúde, 2006.

BRASIL, 2018. Guia de Vigilância Epidemiológica. Departamento de Vigilância Epidemiológica, Secretaria de Vigilância em Saúde, Ministério da Saúde, Brasília, DF.

CAMARGO NEVES,V.L.; et al. Utilização de coleiras impregnadas com deltametrina para o controle da Leishmnaiose Visceral Americana no Estado de São Paulo. Resultados Preliminares. Boletim Epidemiológico Paulista.12: 7-13; 2004.

CAMARGO-NEVES, V.L.; et al. Avaliação da eficácia Lambdacialotrina para o controle de Lutzomyia longipalpis / Evaluation of Lambdacialotrin efficacy for Lutzomyia longipalpis control. Boletim Epidemiológico Paulista, v. 4, n. 43, p. 411, 2007.

CONTI, R. V., et all. Visceral leishmaniasis epidemiologic evolution in timeframes, based on demographic changes and scientific achievements in Brazil. J Vector Borne Dis; 53(2): 99-104, Apr-Jun de 2016.

DEANE, L. M. Leishmaniose visceral no Brasil - estudos sobre reservatórios e transmissores realizados no Estado do Ceará. Rio de Janeiro, Serviço Nacional de Educação Sanitária, 162p. 1956. (Tese da Universidade de São Paulo)

DIAS, J. C. P. Problemas e possibilidades de participação comunitária no controle das grandes endemias do Brazil. Cadernos de Saúde Pública, v. 14, p.19-37, 1998.

LAINSON, R.; RANGEL, E. F. Lutzomyia longipalpis and the eco-epidemiology of American visceral leishmaniasis, with particular reference to Brazil - A Review. Memórias do Instituto Oswaldo Cruz, v. 100, n. 8, p. 811-827, 2005. 
MARTINS, G. A.; THEÓPHILO, C. R. Metodologia da Investigação Científica para Ciências Sociais Aplicadas. 2. ed. São Paulo: Atlas, 2009.

MONTEIRO, S. G., Parasitologia na medicina veterinária. 2. ed. Rio de Janeiro: Roca, 2017.

MENEZES, J. A.; et al.Fatores de risco peridomiciliares e conhecimento sobre leishmaniose visceral da população de Formiga, Minas Gerais. $\underline{\operatorname{Rev}}$ Bras Epidemiol; 19(2): 362-74, Apr-Jun, 2016.

MORAIS, M. H. F; et al. Avaliação das atividades de controle da leishmaniose visceral em Belo Horizonte, Minas Gerais, 2006-2011. Epidemiologia e Serviços de Saúde, v.24, n. 3, p. 485-496, 2015.

NALEVAIKO, P.C. et al. Leishmaniose no Brasil - Importância e perspectivas. PUBVET, Londrina, V. 5, N. 14, Ed. 161, Art. 1088, 2011.

READY, P. D.. Epidemiology of visceral leishmaniasis. Clinical epidemiology. 6. 147-154. 10.2147/CLEP.S44267, 2014.

SALOMÓN, O. D.; FELICIANGELI, M. D.; QUINTANA, M. G.; AFONSO, M. M.; RANGEL, E. F. Lutzomyia longipalpis urbanisation and control. Memórias do Instituto Oswaldo Cruz, v. 110, n. 7, p. 831-846, 2015.

ROMERO, G. A.; et al. O controle de leishmaniose visceral no Brasil: transformar é preciso. Cadernos de Saúde Pública, v. 32, n. 6, 2016.

SANTANA FILHO, F. C.; et al.Recusas de borrifação de imóveis e ocorrência de casos de leishmaniose visceral na Regional Noroeste de Belo Horizonte. Arq. Bras. Med. Vet. Zootec., v.64, n.4, p.899-908, 2012.

SILVA, E. A.; ANDREOTTI, R.; HONER, M. R. Comportamento de Lutzomyia longipalpis, vetor principal da leishmaniose visceral americana, em Campo Grande, Estado do Mato Grosso do Sul. Revista da Sociedade Brasileira de Medicina Tropical, v. 40, n. 4, p. 420-425, 2007.

SILVA, A. M.; et al. Diversidade, distribuião e abundância de flebotomíneos (Deptera: Psychodidae) no Paraná. Revista Public Health - Neotropical entomology 37(2): 209-225- março/abril de 2008.

TAUIL, L. T. Perspectivas de controle de doenças transmitidas por vetores no Brasil. Revista da Sociedade Brasileira de Medicina Tropical 39(3):275-277, mai-jun, 2006.

TAYLOR, M. A.; COOP, R. L.; WALL, R. L., Parasitologia veterinária. 4. ed. Rio de Janeiro: Editora Guanabara Koogan, 2016.

ZUBEN, A. P.; DONALÍSIO, M. R. Dificuldades na execução das diretrizes do Programa de Vigilância e Controle da Leishmaniose Visceral em grandes 
municípios brasileiros.Cad. Saúde Pública, Rio de Janeiro, 32(6):e00087415, jun, 2016. 


\section{APÊNDICE A-}

PROTOCOLO DE RECOMENDAÇÕES SOBRE O MANEJO AMBIENTAL

NOME DO RESPONSÁVEL:

NOME DE QUEM ATENDEU:

ENDEREÇO:

$\mathrm{Na}$ inspeção realizada em que deverão ser sanadas em foram constatadas as irregularidades a seguir, dias.

\section{PROVIDÊNCIAS A SEREM ADOTADAS:}

( ) Varrer ou rastelar folhas e frutos, ensacar e colocar para coleta de lixo;

( ) Recolher fezes de animais, ensacar e colocar para coleta de lixo;

( ) Aparar grama, recolher, ensacar e colocar para coleta de lixo;

( ) Retirar folhas de vasos de plantas, canteiros e jardins, ensacar e colocar para coleta de lixo;

( ) Capinar quintal, recolher, ensacar e colocar para coleta de lixo;

( ) Eliminar ou enterrar adubo orgânico armazenado ou em produção;

( ) Eliminar a criação de porcos e cavalos em área urbana;

( ) Melhorar as condições higiênicas latualizar vacinação de cães e gatos;(posse responsável)

( ) Restringir o número de galinhas, patos, coelhos e outros animais (área urbana);

( ) Podar galhos de árvores.

Outras

recomendações:

IMÓVEL COM PESQUISA ENTOMOLÓGICA

( ) Sim ( ) Não

CLASSIFICAÇÃO:

$1^{\text {a }}$ visita:

Alto Risco ( ) Médio Risco ( ) Baixo ( ) Sem Risco

$2^{\text {a }}$ visita:

Alto Risco ( ) Médio Risco ( ) Baixo ( ) Sem Risco

$3^{\text {a }}$ visita: 
Alto Risco ( ) Médio Risco ( ) Baixo ( ) Sem Risco

CIENTE

LOCAL

I_L_ 


\title{
Apêndice B -
}

\section{TERMO DE CONSENTIMENTO LIVRE E ESCLARECIDO - Entrevista}

\author{
CENTRO DE ENSINO UNIVERSITÁRIO DE BRASÍLIA \\ TERMO DE CONSENTIMENTO LIVRE E ESCLARECIDO - TCLE
}

Este é um convite para você participar da pesquisa: Validação de Protocolo de Manejo Ambiental Para Controle de Lutzomyia longipalpis Em Áreas Endêmicas Para Leishmaniose Visceral, a qual é coordenada por Prof. ${ }^{a}$ Dr. ${ }^{a}$ Rafaella Albuquerque e Silva, e que segue as recomendações da resolução 466/12 do Conselho Nacional de Saúde e suas complementares.

Sua participação é voluntária, o que significa que você poderá desistir a qualquer momento, retirando seu consentimento, sem que isso lhe traga nenhum prejuízo ou penalidade.

Esse trabalho tem como objetivo validar o protocolo de manejo ambiental para controle da população de flebotomíneos no condomínio RK da região administrativa de Sobradinho, Distrito Federal. Caso decida aceitar o convite, você será submetido(a) ao(s) seguinte(s) procedimentos: ter o seu imóvel classificado quanto a parâmetros ambientais favoráveis ao desenvolvimento de vetores; cumprir com as recomendações sobre manejo ambiental fornecidas pelos membros do projeto de pesquisa, sendo seu imóvel reavaliado a cada três meses; e responder a uma entrevista semiestruturada por meio de um questionário com perguntas objetivas e subjetivas sobre aspectos relacionados à prevenção e controle Leishmaniose Visceral (Calazar).

Os riscos envolvidos com sua participação são: desconforto, medo e constrangimento, que serão minimizados através das seguintes providências: esclarecimento sobre a finalidade da pesquisa, garantia de privacidade durante a realização das entrevistas, do sigilo de identidade pessoal e das informações obtidas. Você terá os seguintes benefícios ao participar da pesquisa: a realização de práticas de manejo ambiental para controlar o vetor da Leishmaniose Visceral no seu domicílio e peridomicílio; assim como a participação em atividades de educação e saúde com base nos resultados da entrevista e esclarecimento de dúvidas sobre a Leishmaniose Visceral (Calazar).

Todas as informações obtidas serão sigilosas e seu nome não será divulgado em nenhum momento. Os dados obtidos nessa pesquisa serão consolidados em material impresso e arquivados na sala da profa coordenadora do projeto na instituição proponente por um período de 05 anos e serão obtidos especificamente para os propósitos da pesquisa, tudo sobre a responsabilidade da Prof. ${ }^{a}$ Rafaella Albuquerque e Silva.

Se você tiver algum gasto que seja devido à sua participação na pesquisa, você será ressarcido pela pesquisadora responsável pelo projeto, a Prof. ${ }^{a}$ Rafaella Albuquerque e Silva, caso solicite. Em qualquer momento, se você sofrer algum dano comprovadamente decorrente desta pesquisa, você terá direito a indenização.

Você ficará com uma via deste Termo e toda a dúvida que você tiver a respeito desta pesquisa, poderá perguntar diretamente a: Prof. $^{a}$ Dr. $^{a}$ Rafaella Albuquerque e Silva, no endereço: CEA, Conjunto Antares quadra S. do condomínio RK de Sobradinho, Ou pelo telefone: (61) 98261-6304.

Declaramos que

Dúvidas a respeito da ética dessa pesquisa poderão ser questionadas ao Comitê de Ética em Pesquisa do UniCEUB no endereço SEPN, 707/907 Via W 5 Norte - Asa Norte, DF, 70790-075, no bloco 6 do Centro de ensino universitário de Brasília ou pelo telefone (61) 3966-1383.

\section{Consentimento Livre e Esclarecido}

Declaro que compreendi os objetivos desta pesquisa, como ela será realizada, os riscos e benefícios envolvidos e concordo em participar voluntariamente da pesquisa "Validação de Protocolo de Manejo Ambiental Para Controle de Lutzomyia longipalpis Em Áreas Endêmicas Para Leishmaniose Visceral".

Participante da pesquisa:

Nome Completo:

Assinatura do Participante da Pesquisa

Pesquisador responsável:

Prof $^{a}$. Dra . Rafaella Albuquerque e Silva

Endereço profissional: SEPN, 707/907 Via W 5 Norte - Asa Norte, DF, 70790-075, fone: (61)98261-

6304

Comitê de Ética e Pesquisa: SEPN, 707/907 Via W 5 Norte - Asa Norte, DF, 70790-075 


\section{Apêndice C-}

\section{QUESTIONÁRIO}

1. O que é leishmaniose?

a) É uma doença causada por um protozoário e conhecida popularmente como calazar;

b) É uma doença causada por uma bactéria e conhecida popularmente como salmonelose;

c) É uma doença causada por um vírus e é passada de pessoa para pessoa;

d) Nenhuma das respostas anteriores;

2. Como esta doença é transmitida?

a) A partir da água contaminada com a bactéria;

b) É através da mordida do cão doente;

c) A doença é transmitida pela picada de insetos que são conhecidos por mosquito-palha;

d) A transmissão se dá pelo ar;

3. Como são chamados os insetos que transmitem a LV?

a) Mosquitos da espécie Aedes aegypti;

b) Mosquitos da espécie Anopheles gambie;

C) Flebotomíneos da espécie Lutzomyia longipalpis;

d) Bactéria da espécie Escherichia coli;

4. É possível combater o inseto transmissor?

a) Sim. Especialmente com o apoio da população, no que diz respeito à higiene ambiental (manejo ambiental). Ou seja, por meio de limpeza periódica dos quintais, retirada da matéria orgânica em decomposição (folhas, frutos, fezes de animais e outros entulhos que favoreçam a umidade do solo, locais onde os flebotomíneos se desenvolvem) e destino adequado do lixo orgânico, a fim de impedir o desenvolvimento das formas imaturas dos flebotomíneos; limpeza dos abrigos de animais domésticos, além da manutenção de animais domésticos distantes do domicílio, especialmente durante a noite, de modo a reduzir a atração dos flebotomíneos para o intradomicílio.

b) Não. São insetos de muito difícil controle, não sendo possível nem utilizar inseticidas domiciliares.

c) Sim, são de fácil controle sendo necessário somente a aplicação do tipo ultrabaixo volume (UBV) de inseticidas. Este tipo de aplicação ocorre quando 
a quantidade de insetos está superior ao esperado. Dessa forma, o carro conhecido como Fumacê passa pela cidade, nas áreas com mais casos da doença, e libera o inseticida nas casas.

d) O controle do inseto se faz exclusivamente com o uso de repelentes.

\section{A Leishmaniose tem tratamento em seres humanos?}

a) Apesar de grave, a Leishmaniose tem tratamento para os humanos. Ele é gratuito e está disponível na rede de serviços do Sistema Único de Saúde.

b) Não. Infelizmente não há tratamento para leishmaniose visceral. 


\title{
Apêndice D-
}

\section{TERMO DE CONSENTIMENTO LIVRE E ESCLARECIDO - Entomologia}

\author{
CENTRO DE ENSINO UNIVERSITÁRIO DE BRASÍLIA \\ TERMO DE CONSENTIMENTO LIVRE E ESCLARECIDO - TCLE
}

\section{Esclarecimentos}

Este é um convite para você participar da pesquisa: Validação de Protocolo de Manejo Ambiental Para Controle de Lutzomyia longipalpis Em Áreas Endêmicas Para Leishmaniose Visceral, a qual é coordenada por Prof.a Dr.a Rafaella Albuquerque e Silva.

Sua participação é voluntária, o que significa que você poderá desistir a qualquer momento, retirando seu consentimento, sem que isso lhe traga nenhum prejuízo ou penalidade.

Esse trabalho tem como objetivo validar o protocolo de manejo ambiental para controle da população de flebotomíneos no condomínio RK da região administrativa de Sobradinho, Distrito Federal. Caso decida aceitar o convite, você será submetido(a) ao(s) seguinte(s) procedimentos: permitir que durante o período entre 12 e 24 meses sejam colocadas duas armadilhas luminosas tipo CDC para coleta de flebotomíneos, sendo uma dentro da casa e a outra no peridomicílio, preferencialmente perto de abrigo de animais. As coletas acontecerão durante 3 noites consecutivas mensalmente ou a cada 3 meses.

Os riscos envolvidos com sua participação são: desconforto, medo e constrangimento, que serão minimizados através das seguintes providências: esclarecimento sobre a finalidade da pesquisa, garantia da privacidade e discrição no momento da colocação e retirada das armadilhas, do sigilo da identidade pessoal e das informações obtidas. Você terá os seguintes benefícios ao participar da pesquisa: a realização de práticas de manejo ambiental para controlar o vetor da Leishmaniose Visceral no seu domicílio e peridomicílio; assim como a participação em atividades de educação e saúde com base nos resultados da entrevista e esclarecimento de dúvidas sobre a Leishmaniose Visceral (Calazar).

Todas as informações obtidas serão sigilosas e seu nome não será divulgado em nenhum momento. Os dados obtidos nessa pesquisa serão consolidados em material impresso e arquivados na sala da profa coordenadora do projeto na instituição proponente por um período de 05 anos e serão obtidos especificamente para os propósitos da pesquisa, tudo sobre a responsabilidade da Prof. Rafaella $^{-}$ Albuquerque e Silva.

Se você tiver algum gasto que seja devido à sua participação na pesquisa, você será ressarcido, caso solicite. Em qualquer momento, se você sofrer algum dano comprovadamente decorrente desta pesquisa, você terá direito a indenização.

Você ficará com uma via deste Termo e toda a dúvida que você tiver a respeito desta pesquisa, poderá perguntar diretamente a: Prof.a Dr.a Rafaella Albuquerque e Silva, no endereço: CEA, Conjunto Antares quadra S. do condomínio RK de Sobradinho, Ou pelo telefone: (61) 98261-6304.

Dúvidas a respeito da ética dessa pesquisa poderão ser questionadas ao Comitê de Ética em Pesquisa do UniCEUB Comitê de Ética e Pesquisa: SEPN, 707/907 Via W 5 Norte - Asa Norte, DF, 70790-075 no endereço abaixo ou pelo telefone: (61) 3966-1383.

\section{Consentimento Livre e Esclarecido}

Declaro que compreendi os objetivos desta pesquisa, como ela será realizada, os riscos e benefícios envolvidos e concordo em participar voluntariamente da pesquisa "Validação de Protocolo de Manejo Ambiental Para Controle de Lutzomyia longipalpis Em Áreas Endêmicas Para Leishmaniose Visceral".

Participante da pesquisa:

Nome Completo:

Assinatura do Participante da Pesquisa

Pesquisador responsável:

Profa. Dra. Rafaella Albuquerque e Silva

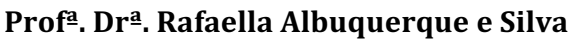

Endereço profissional: SEPN, 707/907 Via W 5 Norte - Asa Norte, DF, 70790-075, fone: (61)98261-6304

Comitê de Ética e Pesquisa: SEPN, 707/907 Via W 5 Norte - Asa Norte, DF, 70790-075 